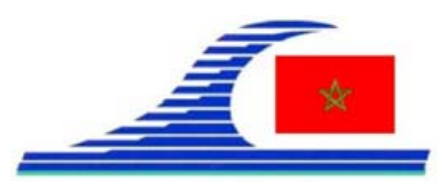

Conférence Méditerranéenne Côtière et Maritime EDITION 2, TANGER, MAROC (2011)

Coastal and Maritime Mediterranean Conference

Disponible en ligne - http://www.paralia.fr - Available online

\title{
Modélisation hydrosédimentaire appliquée à la validation d'un concept innovant de port de plaisance
}

\author{
Martin SANCHEZ ${ }^{1}$, Malek BENAMOUR ${ }^{1}$, Charles ABESSOLO ${ }^{1}$, \\ Georges CLAVERIE $^{2}$, Tom RENAUD ${ }^{3}$, Damien GRIMONT ${ }^{4}$
}

1. Université de Nantes, LPGN, UMR 6112 CNRS, BP 92208, 44322 Nantes, France. martin.sanchez@univ-nantes.fr

2. CREOCEAN, Zone Technocean, rue Charles Tellier, 17000 La Rochelle, France.

3. SCE NANTES, 5 Av Cauchy, BP 10703, 44307 Nantes, France.

4. BLUERING, 5 Av Cauchy, BP 10703, 44300 Nantes, France.

\section{Résumé :}

Le concept de port de plaisance breveté BlueRing est décrit. Ses possibilités d'implantation sont étudiées. Le problème d'envasement est caractérisé en fonction de la turbidité des eaux environnantes et des conditions de marnage de la marée.

Une solution de port autonettoyant est validée à travers une modélisation hydrosédimentaire. Cette solution consiste en une remise en suspension des dépôts de vase effectuée périodiquement à des instants précis, de façon à provoquer une expulsion des sédiments par le courant de jusant.

Enfin, des stratégies pour la gestion des dépôts de vase dans les ports BlueRing sont préconisées selon l'importance du taux d'envasement, et en tenant compte des conditions de marée et de turbidité des eaux environnantes.

\section{Mots-clés :}

Port de plaisance - Port autonettoyant - Port à flot - Port à sec - BlueRing ${ }^{\circledR}$

\section{Introduction}

Cette étude concerne le concept d'aménagement portuaire pour les plaisanciers breveté BlueRing (BLUERING, site web). Il intègre une dimension verticale dans les infrastructures portuaires (figure 1) grâce à l'utilisation de la technique des parois moulées qui sont auto-stables. Cet aménagement est compatible même avec des sols aux qualités géotechniques médiocres, et permet la construction d'ouvrages souterrains profonds et étanches à l'eau, tels que des parkings urbains, des puits d'accès pour tunneliers ou des formes de radoub. Le concept BlueRing a deux applications potentielles :

a) L'implantation d'un port de plaisance en site urbain à densité immobilière élevée sur une surface restreinte de forte valeur foncière. Cette solution permet l'obtention d'un port à flot de prestige en centre-ville et l'aménagement de l'espace souterrain offre une large gamme de possibilités: parking et port à sec (figure 1), surfaces commerciales, bureaux, etc. 
La connaissance de la Mer :

un vecteur du développement durable en Méditerranée

b) L'augmentation de la capacité d'accueil d'un port côtier classique saturé. Dans l'exemple illustré sur la figure 2, l'implantation d'un port BlueRing permet de pratiquement doubler la capacité d'accueil du port tout en conservant constante la surface totale de celui-ci. En effet, dans cet exemple, BlueRing occupe 14\% de la surface totale du port et permet d'accueillir autant de bateaux que le restant du port adjacent.

Un problème récurrent dans les ports de plaisance est celui de l'envasement du bassin, qui est nécessairement abrité de la houle océanique. La possibilité d'inclure une importante partie de port à sec dans les aménagements BlueRing (figure 1) permet de réduire considérablement les dragages calculés en volume de vase rapporté au nombre total d'embarcations.

Ainsi, si l'on prend comme exemple le bassin portuaire illustré sur la figure 2, on constate que pour un même nombre de bateaux abrités, un port BlueRing nécessite un volume de dragages qui est égal à $14 \%$ de celui du secteur portuaire adjacent. Ceci si l'on admet que l'envasement est uniformément reparti sur tout le bassin et que les apports de sédiments se font exclusivement en suspension.

L'objectif de cette étude est de proposer des solutions pour la lutte contre l'envasement dans les ports BlueRing, et ce en fonction :

a) du site d'implantation (port isolé en site urbain ou intégré dans un port côtier) ;

b) des conditions de marée (micro, méso, macro, ou hypertidale) ;

c) des turbidités environnantes (faibles, moyennes, fortes ou très fortes).

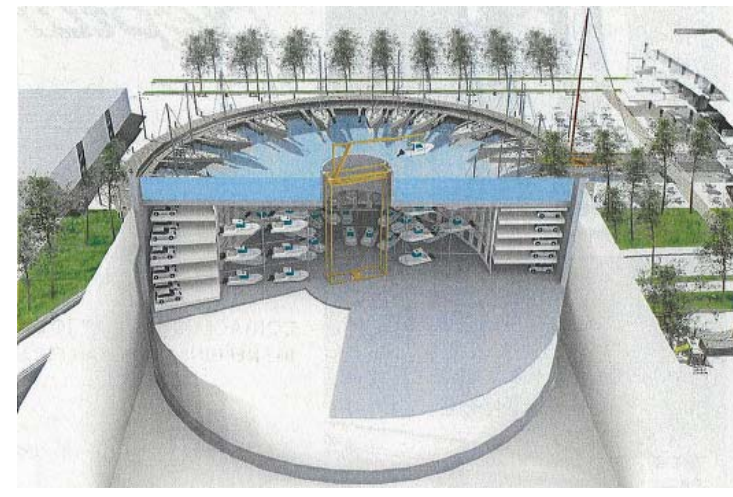

Figure 1. Illustration d'un port à flot en site urbain avec port à sec et parking souterrains, selon le concept BlueRing.

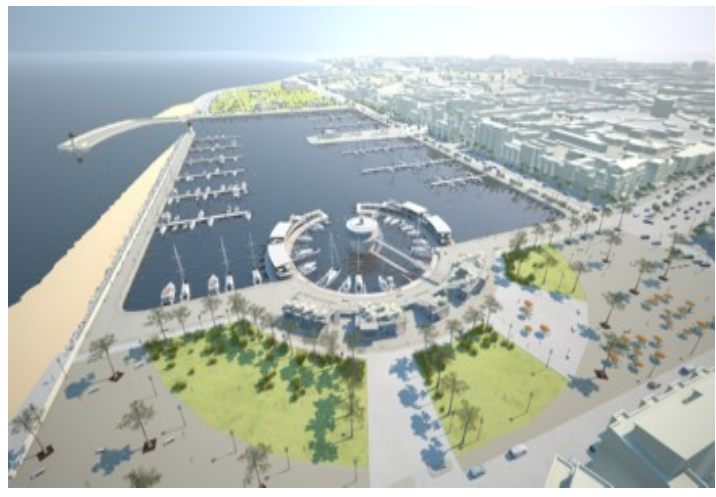

Figure 2. Illustration de l'intégration d'un port de plaisance BlueRing dans un port à flot classique.

\section{Caractérisation de l'envasement selon les facteurs environnants}

L'envasement dans les bassins des ports est engendré en grande partie par les conditions locales d'eau calme. Ces conditions sont à l'origine d'un important dépôt des matières en suspension. L'envasement dépend ainsi des facteurs suivants : 
a) Volume d'eau oscillant par cycle de marée.

b) Turbidité ou teneur en matières en suspension dans les eaux environnantes.

c) Caractéristiques des matières en suspension dont la vitesse de chute.

L'ordre de grandeur de l'envasement dans un bassin à flot peut être établi à travers l'étude du bilan sédimentaire sur un cycle de marée complet comme suit :

Vase entrant dans le port = Vase sortant du port + Vase déposée dans le port

En première approximation sur un cycle de marée on peut évaluer :

Vase entrant du port $=C_{e} S H$

Vase sortant du port $=C_{p} S H$

Vase déposée dans le port $=C_{p} W^{\text {ef }} S T$

où $C_{e}$ est la concentration moyenne en matières en suspension (MES) dans l'eau environnante, $C_{P}$ la concentration moyenne en MES dans l'eau du port, $S$ la surface horizontale du bassin portuaire, $H$ le marnage, $T$ la période de la marée, et $W^{e f}$ la vitesse de chute effective des MES dans le port qui peut être calculée par la formulation d'Owen suivante :

$W^{\text {ef }}=A C_{p}{ }^{B}$

où $A$ et $B$ sont deux paramètres spécifiques à chaque vase étudiée. Le terme $C_{p}$ est relié à $C_{e}$ par l'expression suivante :

$C_{p}=\frac{H}{H+W^{e f} T} C_{e}$

L'envasement dans le port par unité de surface horizontale sur un cycle de marée est donné par le produit $C_{p} \times W^{e f} \times T$. L'envasement annuel est obtenu en multipliant ce produit par le nombre de cycles de marée dans une année. Enfin, le taux d'envasement en $\mathrm{m} \mathrm{an}^{-1}$ est obtenu en divisant l'envasement annuel par la concentration de la vase déposée.

La figure 3 reliant le taux d'envasement à la concentration en MES dans l'eau environnante, pour 4 différentes valeurs du marnage moyen Hmoy, a été dressée pour une marée semi-diurne de période $12 \mathrm{~h} 24$ ', une concentration de la vase déposée de $300 \mathrm{~kg} \mathrm{~m}^{-3}$, et des paramètres de la formulation d'Owen $A=0,002 \mathrm{~m} \mathrm{~s}^{-1}$ et $B=1$ (avec $W^{e f}$ donné en $\mathrm{m} \mathrm{s}^{-1}$ et $C_{p}$ en $\mathrm{kg} \mathrm{m}^{-3}$ ) sélectionnés selon des valeurs préconisées dans la littérature spécialisée (DHI, 2007). Les valeurs des marnages étudiés sont les suivantes :

a) $H$ moy=8,00 m, Hmax $\approx 13,2 \mathrm{~m}$ - marée hypertidale.

b) $H$ moy $=3,60 \mathrm{~m}, H \max \approx 6,2 \mathrm{~m}$ - marée macrotidale.

c) $H$ moy $=1,20 \mathrm{~m}, H \max \approx 2,1 \mathrm{~m}$ - marée mésotidale.

d) $H$ moy $=0,30 \mathrm{~m}, H \max \approx 0,5 \mathrm{~m}$ - marée microtidale.

Bien que le taux d'envasement évalué soit dépendant des valeurs retenues des paramètres de la loi d'Owen, on peut indiquer que les courbes de la figure 3 restent valables en première approximation pour toutes les vases marines et estuariennes. 
La connaissance de la Mer :

un vecteur du développement durable en Méditerranée

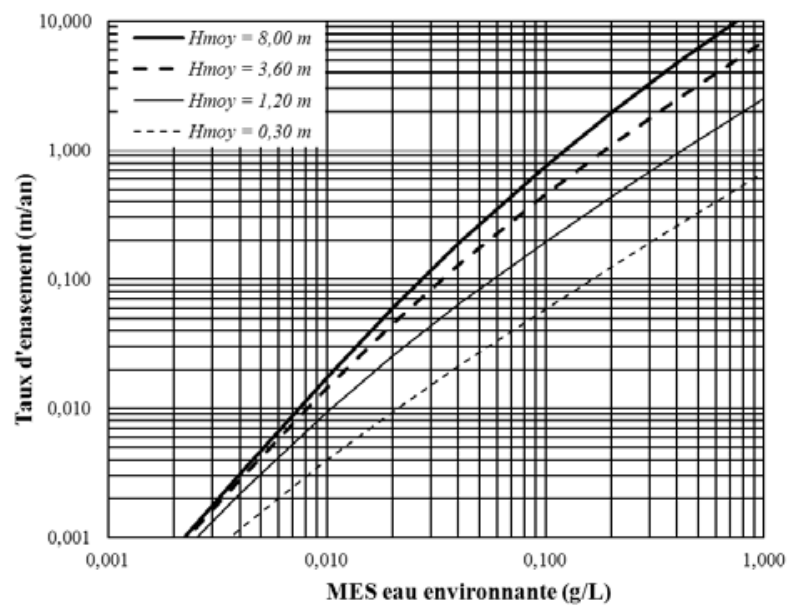

Figure 3. Taux d'envasement en fonction de la concentration en MES dans l'eau environnante pour 4 valeurs du marnage moyen Hmoy

(pour une marée semi-diurne et $W^{\text {ef }}=0,002 C_{p}$, avec $W^{\text {ef }}$ en $\mathrm{m} \mathrm{s}^{-1}$ et $C_{p}$ en $\mathrm{kg} \mathrm{m}^{-3}$ ).

\section{Gestion de l'envasement selon les cas étudiés}

Selon le lieu d'implantation d'un port BlueRing, trois cas de sédimentation sont susceptibles de se présenter (SANCHEZ et al., 2010) : (i) dépôts de sables, (ii) dépôts de mélanges sable-vase, et (iii) dépôts de vases seules.

Les deux premiers cas peuvent être envisagés quand le projet BlueRing s'intègre dans un port côtier classique (figure 2). La gestion et la valorisation des sédiments doit se faire conjointement avec ceux des zones adjacentes par les méthodes usuelles.

Le troisième cas est envisagé quand le projet BlueRing est isolé du domaine océanique (figure 1). En effet, sachant que les courants à travers le canal d'accès seront en général faibles, le potentiel de transport de sables par charriage sera pratiquement nul.

Par ailleurs, pour un port BlueRing isolé du domaine océanique, le taux d'envasement dépend principalement du marnage de la marée et de la turbidité de l'eau environnante comme il est montré sur la figure 3. La turbidité varie d'une façon importante d'une région à une autre :

a) $C_{e}<0,002 \mathrm{~kg} \mathrm{~m}^{-3}$ : eau océanique transparente de grandes profondeurs.

b) $0,002<C_{e}<0,01 \mathrm{~kg} \mathrm{~m}^{-3}$ : eau océanique du plateau continental.

c) $0,01<C_{e}<0,05 \mathrm{~kg} \mathrm{~m}^{-3}$ : eau des régions côtières exposées à la houle.

d) $0,05<C_{e}<0,2 \mathrm{~kg} \mathrm{~m}^{-3}$ : eau des régions abritées à très forte turbidité (baies et estuaires).

e) $C_{e}>0,2 \mathrm{~kg} \mathrm{~m}^{-3}$ : turbidité de pointe dans les régions à forte turbidité (bouchon vaseux).

En ce qui concerne le marnage, les cas les plus défavorables correspondent aux marées hypertidale et macrotidale :

a) L'implantation d'un port BlueRing dans un site soumis à une marée hypertidale peut nécessiter la mise en place d'une écluse afin de conserver un plan d'eau quasiment fixe correspondant à un niveau de pleine mer. Le blocage du volume d'eau oscillant par la présence de l'écluse règle aussi le problème de l'envasement. 
b) L'implantation d'un port BlueRing dans un site soumis à une marée macrotidale peut nécessiter la mise en place d'une porte limitant le volume d'eau oscillant de 50\%, réduisant d'autant le taux d'envasement. En effet, usuellement les portes d'accès aux ports sont maintenues fermées pendant tout le demi-cycle de marée au cours duquel le niveau de la mer est inférieur à celui de mi-marée.

\section{Le port BlueRing autonettoyant}

On constate que le problème d'envasement est d'autant plus important, que le marnage est élevé (tous les autres paramètres du problème étant conservés constants). Cependant, la marée représente aussi une énergie pouvant être utilisée pour évacuer les vases déposées. Ainsi, une solution retenue pour parvenir à la mise en place d'un port autonettoyant consiste en une remise en suspension mécanique des vases fraîchement déposées sur le fond rigide en béton des ports BlueRing, de manière à ce que les sédiments soient évacués pendant le jusant.

Une série de simulations numériques a été effectuée à l'aide du logiciel Mike 21 (DHI, 2007) afin de valider le concept de port autonettoyant (BENAMOUR, 2011). Les résultats présentés par la suite correspondent à une marée macrotidale (Hmoy=3,6 m) dans une région à très forte turbidité $\left(C_{e}=0,2 \mathrm{~kg} \mathrm{~m}^{-3}\right)$. L'action mécanique entraînant la remise en suspension est caractérisée par une contrainte tangentielle $\tau$ agissant au fond pendant un durée $2 \Delta t=1800 \mathrm{~s}$. Pendant un premier laps de temps $\Delta t$ la valeur de $\tau$ varie linéairement de 0 à $5,5 \mathrm{~N} \mathrm{~m}^{-2}$ et pendant un deuxième laps de temps de même durée $\Delta t$ la valeur de $\tau$ passe de 5.5 à $0 \mathrm{~N} \mathrm{~m}^{-2}$. La figure 4 montre l'évolution de l'envasement calculé à la basse mer $(\mathrm{BM})$ en fonction du nombre de cycles de marée. On constate qu'en l'absence de remise en suspension après 10 cycles l'envasement cumulé est d'environ $4,4 \mathrm{~kg} \mathrm{~m}^{-2}$, ce qui permet d'extrapoler à $310 \mathrm{~kg} \mathrm{~m}^{-2} \mathrm{an}^{-1}$, correspondant bien aux résultats de la figure 3 obtenus par une autre méthode, sachant que la valeur retenue pour la concentration de la vase déposée est de $300 \mathrm{~kg} \mathrm{~m}^{-3}$.

La solution d'un port autonettoyant correspond aux asymptotes horizontales des courbes montrées sur la figure 4 (taux d'envasement nul obtenu par un équilibre entre la masse d'eau entrant et sortant du port). On constate que la meilleure solution est obtenue quand la remise en suspension s'effectue entre 1 et 3 heures après la pleine mer. Enfin, on doit signaler qu'un port autonettoyant peut être obtenu par une remise en suspension journalière, hebdomadaire ou mensuelle, quand les taux d'envasement sont moins importants que dans le cas extrêmement défavorable ici étudié.

\section{Conclusions et perspectives}

Les solutions qui peuvent être préconisées pour la gestion des dépôts de vase dépendent de leur importance :

a) Taux d'envasement de l'ordre de 0,01 $\mathrm{m} \mathrm{an}^{-1}$ : un entretien décennal peut être envisagé par des moyens mécaniques ou hydrauliques (drague aspiratrice). 
La connaissance de la Mer :

un vecteur du développement durable en Méditerranée

b) Taux d'envasement de l'ordre de $0,1 \mathrm{~m} \mathrm{an}^{-1}$ : un entretien annuel par dragage peut être envisagé ou une remise en suspension mécanique mensuelle des dépôts.

c) Taux d'envasement de l'ordre de $1 \mathrm{~m} \mathrm{an}^{-1}$ : un port auto-nettoyant avec une remise en suspension mécanique journalière peut être mis en place (en complément d'une porte dans le cas d'une marée macrotidale).

d) Taux d'envasement supérieur à $2 \mathrm{~m} \mathrm{an}^{-1}$ : ce cas, concernant essentiellement les sites soumis à une marée hypertidale dans les zones à forte turbidité, justifie la mise en place d'une écluse.

La mise en œuvre d'un port BlueRing autonettoyant nécessite l'utilisation d'un dispositif mobile pour la remise en suspension de vases déposées dont la conception fait l'objet d'une étude en cours.

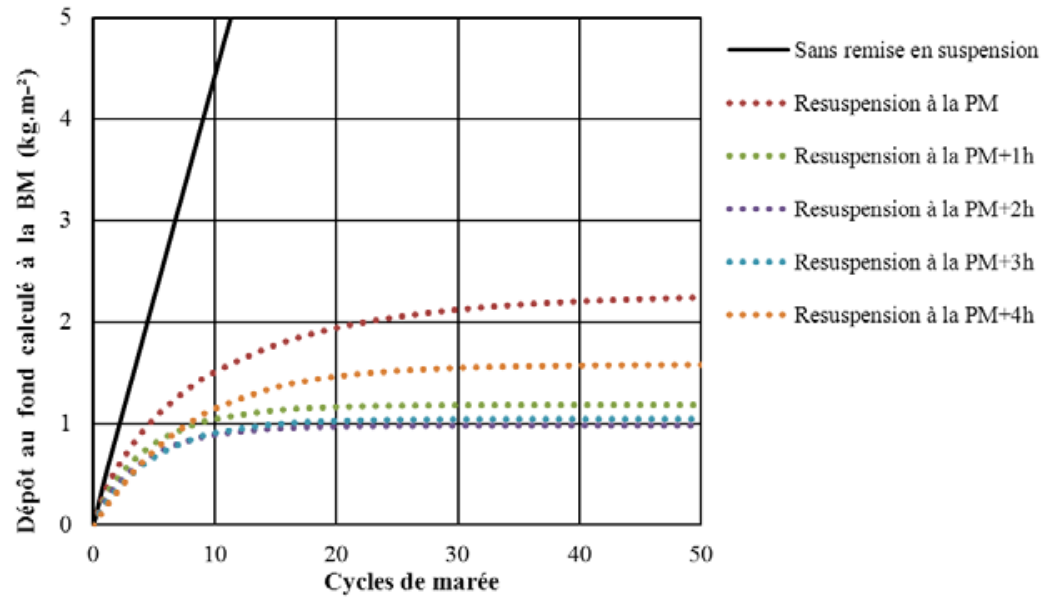

Figure 4. Résultats de la modélisation hydrosédimentaire visant la validation d'un concept de port autonettoyant, montrant le dépôt de vase calculé à la basse mer.

Remerciements : Les auteurs remercient le Pôle Génie Civil Ecoconstruction qui a labellisé ce projet collaboratif, ainsi que les financeurs à travers le Fonds Unique Interministériel, la Région Pays de la Loire, et le Conseil Général de l'Ain.

\section{Références}

BLUERING (site web). http://www.bluering.fr/

BENAMOUR M. (2011). Validation d'un système d'auto-devasement dans un port de plaisance par une modélisation hydrosédimentaire 2D. Mémoire de Master Ingénierie et Géosciences du Littoral, Université de Caen Basse-Normandie, 37 p.

DHI (2007). MIKE 21 \& MIKE 3 flow model FM. Hørsholm, Denmark, 14 p.

SANCHEZ M., ABESSOLO C., GRIMONT D., COMY D., RENAUD T., ALESSANDRINI B., DROUET A., CLAVERIE G. (2010). Ingénierie hydrosédimentaire appliquée à la validation d'un concept innovant de port de plaisance. JNGCGC, Les Sables d'Olonne, pp 937-944. doi:10.5150/jngcgc.2010.102-S 